\title{
EELS Studies of Extrinsic Multiferroics
}

\author{
B.Warot-Fonrose, E.Snoeck, G.Delaizir, P.Rozier, M.Dollé, V. Serin and J.F. Bobo
}

CEMES-CNRS, 29 rue Jeanne Marvig, 31055 - Toulouse, France

Multiferroic materials present two properties among ferroelectricity, ferromagnetism and ferroelasticity [1]. The growth of such materials should allow the control of one of this property through the tuning of another one. It could for example be possible to control the magnetic properties of a multiferroic thin layer through the application of an electric field [2]. Multiferroic materials are separated in two categories: the intrinsic and extrinsic ones. $\mathrm{BiFeO}_{3}$ [3] is ferroelastic and antiferromagnetic and belongs to the first class. The symmetry of the $\mathrm{BiFeO}_{3}$ cell and the tilt of oxygen octaedra are two key parameters to understand the physical behavior of this material [4]. The second class corresponds to hybrid systems combining materials with different ferroic properties. The interaction between the two materials could be complex but the interfaces play a key role in the resulting multiferroic properties [5,6]. Much effort has been dedicated to this subject with both theoretical and experimental studies. Surprisingly, only few studies deal with chemical and structural characterization of interfaces.

In this study, extrinsic multiferroic compounds composed by the ferrimagnetic $\mathrm{CoFe}_{2} \mathrm{O}_{4}$ material associated to the ferroelastic - ferroelectric $\mathrm{PbTiO}_{3}$ oxide are synthesized using the Spark Plasma Sintering System . The resulting high density ceramic is polycrystalline as shown on figure 1. VSM experiments reveal a jump in the $\mathrm{CoFe}_{2} \mathrm{O}_{4}$ magnetization at the $\mathrm{PbTiO}_{3}$ Curie temperature indicating a coupling between the $\mathrm{CoFe}{ }_{2} \mathrm{O}_{4}$ magnetization and the $\mathrm{PbTiO}_{3}$ ferrolectricity.

This work will propose EDX and EELS studies of the interfaces between the two oxides and will correlate the local chemical information with magnetic measurements. Figure 2 presents a typical EEL spectrum obtained on a large area of the thin film, showing edges of interest for chemical mapping at the interface.

\section{References}

[1] L.W..Martin et al., Materials Science and Engineering R 68 (2010) 89-133

[2] V. Laukhin, et al., Phys. Rev. Lett. 97 (2006) 227201

[3] H. Béa et al., Phys. Rev. Letters 102 (2009) 217603

[4] A.Y. Borisevich et al., ACS Nano 4 (2010) 6071

[5] H. Zheng, F. et al., Advanced Materials 18 (2006) $202747-2752$

[6] N. Dix et al, Appl. Phys. Lett. 95 (2009) 062907 


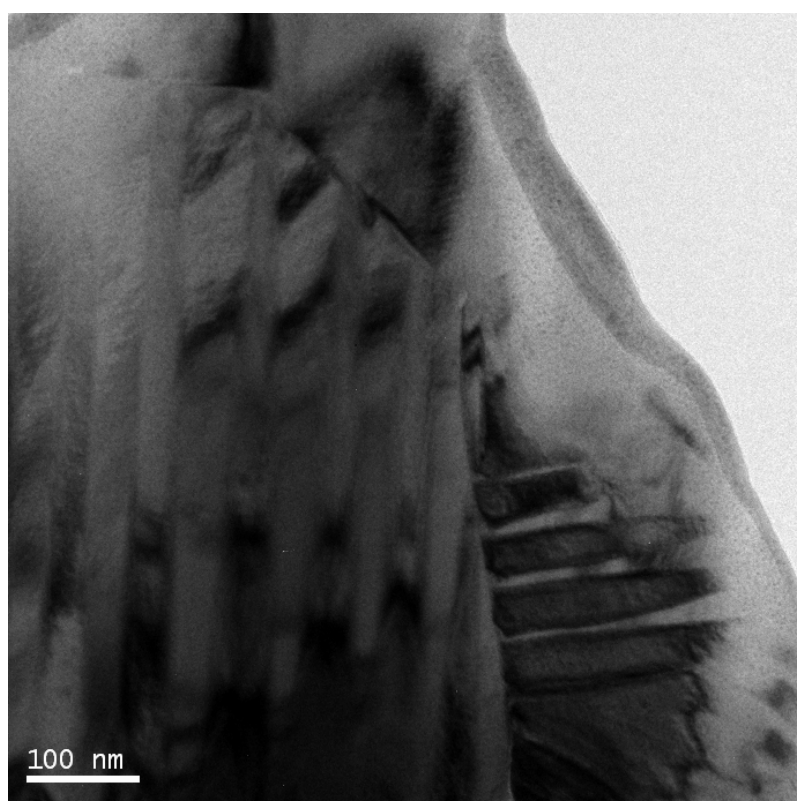

FIG. 1. TEM micrograph of the extrinsic multiferroic material with contrasts arising from the ferroelectric regions

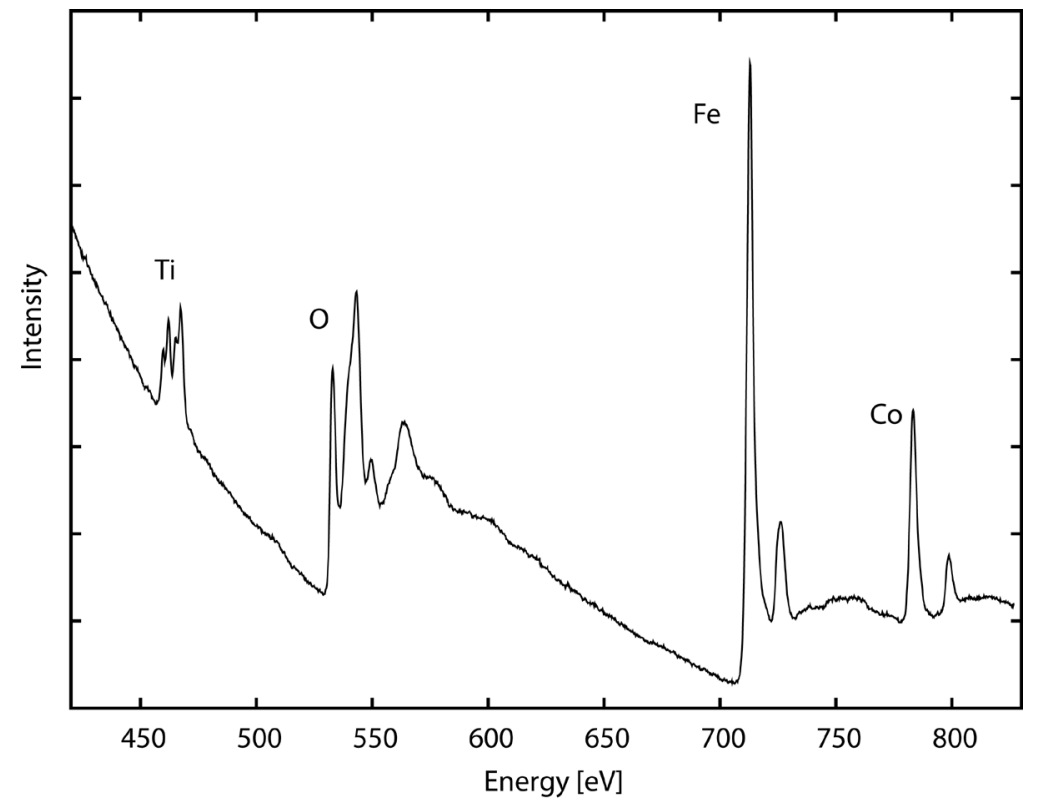

FIG. 2. EEL spectrum obtained on several grains of the samples. The edges in the spectrum will be used to map the chemistry at the interface. 